\title{
Inégalités de santé associées à la défavorisation du secteur de résidence au sein de la population du Québec ayant reçu un diagnostic d'hypertension artérielle en prévention primaire des maladies cardiovasculaires
}

A. Vanasse, M.D., Ph. D. (1, 2); J. Courteau, Ph. D. (2); S. Asghari, M.D., Ph. D. (3); D. Leroux, Ph. D. (4); L. Cloutier, Ph. D. (5)

Cet article a fait l'objet d'une évaluation par les pairs.

Diffuser cet article sur Twitter

\section{Résumé}

Introduction : Bien que plusieurs études traitent de la prévalence, de l'incidence, du traitement, de la mortalité et de la morbidité en lien avec l'hypertension artérielle (HTA), peu d'entre elles tiennent compte de l'influence des secteurs de résidence sur ces indicateurs de santé au sein de la population ayant reçu un diagnostic d'HTA.

Objectifs : L'objectif de cette étude était de mesurer et comparer la prévalence, la mortalité, la morbidité, l'utilisation des ressources médicales et les traitements selon le niveau de défavorisation matérielle et sociale du secteur de résidence au sein de la population ayant reçu un diagnostic d'HTA en prévention primaire des maladies cardiovasculaires (MCV) au Québec en 2006-2007.

Méthodologie : Cette étude repose sur une analyse secondaire des données médicoadministratives de la Régie de l'assurance maladie du Québec pour une cohorte de 276793 patients de 30 ans et plus ayant reçu un diagnostic d'HTA en 2006 ou en 2007 mais sans diagnostic connu de MCV. Les indicateurs de santé ajustés pour l'âge et le sexe sont la prévalence, le décès, un événement cardiovasculaire, les consultations médicales, les consultations à l'urgence ainsi que l'utilisation d'antihypertenseurs. Vingt-cinq types de secteurs de résidence ont été obtenus par croisement des quintiles de défavorisation matérielle et sociale.

Résultats : Par rapport aux patients vivant dans des secteurs favorisés matériellement et socialement, ceux vivant dans des secteurs défavorisés avaient $46 \%$ plus de risque d'événement cardiovasculaire, 47 \% plus de risque d'être de grands utilisateurs de l'urgence, $31 \%$ plus de risque d'être de grands utilisateurs des services d'un omnipraticien, mais $25 \%$ moins de risque d'être de grands utilisateurs des services de médecins spécialistes. Peu ou pas de variation n’a été observée quant à l'utilisation d'antihypertenseurs.

Conclusion : Cette étude révèle l'existence, dans le cadre de la prévention primaire des $\mathrm{MCV}$, d'importantes variations pour plusieurs indicateurs de santé chez des patients hypertendus en raison de la défavorisation matérielle et sociale de leur secteur de résidence. Il est donc important de tenir compte du contexte socioéconomique lors de la planification d’interventions visant à prévenir les maladies cardiovasculaires et leurs conséquences.

Mots-clés : défavorisation matérielle, défavorisation sociale, grands utilisateurs de services, hypertension artérielle, indicateur de santé, maladies cardiovasculaires, régions métropolitaines, régions rurales

\section{Introduction}

L'hypertension artérielle (HTA) est un problème de santé publique important : c'est un facteur de risque de maladie cardiovasculaire (MCV) et d'insuffisance rénale ainsi qu'un facteur de risque de mortalité, parmi les plus importants en termes d'années de vie corrigées de l'incapacité ${ }^{1-3}$. Kearney et collab. ${ }^{4}$ ont estimé la prévalence globale de l'HTA, généralement définie comme étant une tension artérielle systolique égale ou supérieure à $140 \mathrm{mmHg}$ ou une tension artérielle diastolique égale ou supérieure à $90 \mathrm{mmHg}$, à $26 \%$ de la population adulte en 2000 et s'attendent en 2025 à une augmentation de $24 \%$ dans les pays développés et de $80 \%$ dans les pays en développement. L'étude de Danaei et collab. ${ }^{5}$ fait plutôt état d'une baisse générale de la tension artérielle (TA) systolique moyenne à travers le monde pour les deux sexes, avec une prévalence de l'hypertension (ajustée pour l'âge) passant de $33 \%$ en 1980 à $29 \%$ en 2008 chez les hommes et de $29 \%$ à $25 \%$ chez les femmes. Cependant, cette tendance est très variable selon les pays et les régions. De plus, malgré cette tendance à la baisse, le nombre absolu de personnes souffrant d'hypertension a augmenté à cause du vieillissement de la population et de la croissance de la population mondiale.

Rattachement des auteurs :

1. Département de médecine de famille et de médecine d'urgence, Université de Sherbrooke, Sherbrooke (Québec), Canada 2. Groupe de recherche PRIMUS, Centre de recherche CHUS, Sherbrooke (Québec), Canada

3. Primary Healthcare Research Unit, Faculty of Medicine, Health Sciences Centre, St. John's (Terre-Neuve-et-Labrador), Canada

4. Département de géographie, Université du Québec à Trois-Rivières (UQTR), Trois-Rivières (Québec), Canada

5. Département des sciences infirmières, Université du Québec à Trois-Rivières (UQTR), Trois-Rivières (Québec), Canada

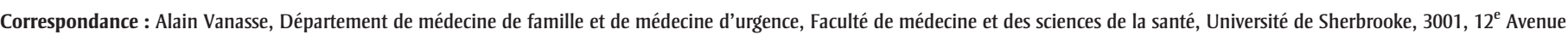
Nord, Sherbrooke (Québec) J1H 5N4; tél. : 819-346-1110, poste 16641; courriel : alain.vanasse@usherbrooke.ca 
D'après une étude ontarienne publiée en 2008, $21 \%$ de la population de 20 à 79 ans souffrait d'HTA en $2006^{6}$ et, d'après les résultats de l'Enquête canadienne sur les mesures de la santé (ECMS) pour 2007$2009^{7}, 19 \%$ des Canadiens de 20 à 79 ans étaient atteintes d'HTA et $20 \%$ avaient une TA les classant dans la fourchette de la préhypertension. La prévalence d'HTA demeure cependant plus faible au Canada qu'aux États-Unis (29\%) et en Angleterre $(30 \%)^{8}$.

Les résultats du suivi de l'HTA réalisé par l'Institut national de la santé publique du Québec (INSPQ) ${ }^{9-10}$ indiquent qu'au Québec la prévalence relative ajustée est passée de $15,8 \%$ en 2000 à $20,3 \%$ en 2007, avec une progression plus rapide chez les hommes. Dans le même temps, le taux ajusté d'incidence de l'hypertension a diminué, et ce, pour les deux sexes. Les taux de mortalité chez les personnes souffrant d'hypertension ont également diminué entre 2000 et 2007 pour les deux sexes, peut-être grâce à l'accès à de meilleurs traitements pharmacologiques au fil des années ${ }^{9-10}$. Par ailleurs, une étude récente ${ }^{11}$ effectuée dans 17 pays a évalué la prévalence de l'HTA à près de $40 \%$ de la population de 35 à 70 ans, la définition de l'HTA reposant sur l'utilisation (autodéclarée) d'un antihypertenseur ou sur une tension artérielle (TA) moyenne d'au moins 140/90 $\mathrm{mmHg}$ ( 2 mesures). Moins de la moitié (46,5\%) des participants à cette étude étaient au courant de leur état et seulement le tiers $(32,5 \%)$ de ceux qui suivaient un traitement avaient une TA contrôlée.

Selon l'Organisation mondiale de la Santé $(\mathrm{OMS})^{12}$, une combinaison de facteurs, ou déterminants de la santé, a un effet sur la santé d'un individu. Dans le modèle de l'OMS $^{12}$ ces déterminants sont classés en trois catégories : ceux liés aux caractéristiques individuelles des patients (âge, sexe ou comorbidité par exemple), ceux liés aux caractéristiques sociales et physiques du secteur de résidence (niveau socioéconomique ou caractère rural par exemple) et ceux liés aux caractéristiques du système de soins de santé et des pratiques de soins ${ }^{13-16}$. Les caractéristiques des secteurs de résidence peuvent en effet influencer certains comportements, notamment les habitudes alimentaires et l'activité physique ${ }^{17}$, ce qui peut avoir des répercussions sur la santé ${ }^{18}$. Les facteurs susceptibles d'avoir une incidence sur la santé sont entre autres la qualité des logements abordables et leur disponibilité $^{19}$, la pauvretée ${ }^{20}$, la sécurité ${ }^{21}$ ou encore le climat social chaleureux et la cohésion qui caractérisent un secteur bien organisé et un tissu social serré22.

Le lien entre les caractéristiques du milieu de vie (niveau socioéconomique ou caractère urbain/rural du secteur) et la prévalence et l'incidence de l'HTA a déjà été étudié. Les résultats de l'étude de Chow et collab. ${ }^{11}$, publiés en 2013, montrent par exemple que la conscience que les participants de l'étude avaient de leur état hypertensif, leur traitement de même que le contrôle de leur TA étaient plus élevés dans les collectivités urbaines que rurales dans les pays à faibles revenus. Cependant, la conscience, le traitement et le contrôle étaient similaires dans les collectivités urbaines et rurales dans les pays à plus hauts revenus. De plus, le contrôle de la TA était plus fréquent dans les pays à hauts revenus $(40,7 \%)$. Lee et collab. ${ }^{23}$ ont constaté l'existence d'un gradient entre la prévalence de l'hypertension artérielle et le revenu, tandis qu'Aubé-Maurice et collab. ${ }^{24}$ ont montré que l'incidence de l'hypertension artérielle était associée à la défavorisation matérielle et sociale du secteur, cette association différant cependant selon l'algorithme d'identification des cas.

En résumé, bien que l'association entre le niveau de défavorisation (individuel, d'un secteur ou d'un pays) et les indicateurs de santé comme la prévalence et l'incidence de l'HTA soient bien documentés, peu d'études portent sur l'influence des caractéristiques du secteur de résidence sur la mortalité, la morbidité, l'utilisation des services de santé et le traitement médicamenteux pour une population ayant reçu un diagnostic d'HTA en prévention primaire des MCV. De surcroît, plusieurs des enquêtes actuelles n'ont pas la puissance statistique adéquate pour bien évaluer les populations vulnérables $^{25}$, par conséquent nous avons tenté de répondre aux questions suivantes :

- Dans cette population vulnérable, eston plus à risque de mortalité et de morbidité dans les secteurs défavorisés?

- Qui sont les plus grands utilisateurs de services de première ligne et de deuxième ligne?

- Est-ce que les personnes vivant dans les secteurs défavorisés sont moins souvent traitées pour leur hypertension artérielle que celles vivant dans les secteurs moins défavorisés?

- Observe-t-on des différences entre les régions métropolitaines et non métropolitaines?

Les objectifs de cette étude étaient de décrire et de comparer, selon le niveau de défavorisation matérielle et sociale du secteur de résidence de la population, la prévalence de l'HTA en prévention primaire de MCV en 2006-2007 au Québec, de même que, pour les personnes souffrant d'HTA, la mortalité, la morbidité, l'utilisation des soins médicaux et les traitements médicamenteux. Puisque la défavorisation matérielle et sociale du secteur diffère généralement selon le milieu de vie (urbain ou rural), les comparaisons ont été réalisées à la fois globalement et par type de milieu (ici région métropolitaine ou non).

\section{Méthodologie}

\section{Sources des données}

Il s'agit d'une analyse secondaire des données médico-administratives de la Régie de l'assurance maladie du Québec (RAMQ) ${ }^{26}$ provenant du fichier des bénéficiaires, du fichier des services rémunérés à l'acte ainsi que du Fichier des hospitalisations Med-Écho. Ce dernier contient les diagnostics, les dates d'admission et de sortie de l'hôpital ainsi que tous les actes thérapeutiques effectués ${ }^{27}$. Le fichier des services rémunérés à l'acte contient le numéro encrypté du médecin traitant, l'acte posé, le diagnostic et la date du service. Le fichier des bénéficiaires indique le sexe, la date de naissance et la localisation géographique du lieu de résidence (code postal). Le fichier des décès, fourni par l'Institut de la statistique du Québec, indique la date et la cause de tous les décès se produisant au Québec. D’autres données ont été fournies par la RAMQ par l'entremise du fichier des 
professionnels de la santé (numéro encrypté du médecin, spécialité du médecin), du fichier d'admissibilité au régime public d'assurance médicament (dates de début et de fin de participation) et du fichier sur les services pharmaceutiques facturés par les pharmaciens à la RAMQ (qui contient toutes les demandes de remboursement de médicaments faites par les personnes couvertes par le régime public, avec le code du médicament, la date de réclamation et la durée du traitement).

En ce qui concerne les médicaments, la RAMQ couvrait environ $41 \%$ de la population en 2006, à savoir les personnes de 65 ans et plus, les bénéficiaires de l'aide sociale ainsi que toutes les personnes non couvertes par un régime privé d'assurance médicament. L'aire de diffusion* (AD) correspondant au secteur de résidence a été associée à chaque patient à l'aide du code postal de sa résidence ${ }^{28}$. Les données sur les indices de défavorisation matérielle et sociale proviennent de l'INSPQ ${ }^{29}$. La classification des $\mathrm{AD}$ en fonction de l'appartenance à une région urbaine, une petite ville ou une région rurale a été effectuée à l'aide des données fournies par Statistique Canada ${ }^{28}$. Les données sur les effectifs de population par AD en fonction de l'âge et du sexe sont basées sur le recensement de 2006 et ont été fournies par Statistique Canada ${ }^{30}$. Ce projet a obtenu l'approbation du Comité d'éthique de l'Université de Sherbrooke et de la Commission d'accès à l'information du Québec.

\section{Population}

La cohorte à l'étude est constituée de toutes les personnes de 30 ans et plus résidant au Québec, ayant été hospitalisées avec un diagnostic primaire ou secondaire d'HTA conforme à la Classification internationale des maladies (CIM-9 : 401 ou CIM-10 : I10) ou ayant utilisé au moins trois services médicaux en 365 jours avec un diagnostic d'HTA durant la période à l'étude (janvier 2006 à décembre 2007). Bien que d'autres algorithmes aient été validés ${ }^{31}$ et aient présenté à la fois une bonne sensibilité et une bonne spécificité, ils n’ont pas été retenus car ils faisaient intervenir des données sur le dossier médical et sur les médicaments, et nous n'avions pas accès au dossier médical et au fichier sur les médicaments pour tous les patients. L'algorithme de définition de cas d'HTA se rapprochant le plus du nôtre a été validé par Lix et collab. ${ }^{32}$ sur les données du Manitoba ( 1 hospitalisation ou 2 services en 1 an). Il offrait une sensibilité de $51 \%$ et une spécificité de $97 \%$.

Pour ne conserver que les patients en prévention primaire des MCV, ont été exclus de la cohorte ceux ayant été hospitalisés avec un diagnostic de MCV, à savoir une cardiopathie ischémique (CIM-9 : 410-414 ou CIM-10 : I20-I25), de l'insuffisance cardiaque (CIM-9 : 428 ou CIM-10 : I50) ou une maladie vasculaire cérébrale (CIM-9 : 430-438 ou CIM-10 : I60-I69), dans les 4 ans précédant la date de référence (la première date pour laquelle une HTA a été diagnostiquée durant la période à l'étude constitue la date de référence). Ont été exclues également toutes les patientes ayant consulté ou ayant été hospitalisées pour une grossesse (CIM-9 : 630-676 et 760-779 ou CIM-10 : O00-099 et Z32-Z39) dans les 5 mois suivant la date de référence ${ }^{33}$. Enfin, ont été également exclus les patients pour lesquels aucune $\mathrm{AD}$ ne pouvait être définie ou aucune information sur la taille de la population de leur $\mathrm{AD}$ n'était disponible, ainsi que ceux relevant d'AD dont la valeur de défavorisation matérielle et sociale était inconnue.

\section{Variables}

La première variable à l'étude est la prévalence de l'HTA en prévention primaire des MCV, en fonction de la défavorisation matérielle et sociale. Pour chaque unité sociogéographique retenue, la prévalence est composée au numérateur de la taille de la cohorte à l'étude et au dénominateur de la taille de la population du Québec âgée de 30 ans et plus.

On a calculé également l'incidence de la mortalité toutes causes confondues et celle d'un événement cardiovasculaire donné (mortalité par MCV ou hospitalisation pour une MCV; CIM-9 : 410-414, 428 et 430-438; CIM-10 : I20-I25, I50 et I60-I69) pour la totalité de la cohorte sur les deux ans suivant la date de référence. Pour les autres variables dépendantes, les incidences ont été calculées pour les personnes ayant survécu au terme de la période de deux ans suivant la date de référence. Ces variables sont : une hospitalisation toutes causes confondues, une consultation ambulatoire (pour tout problème de santé) d'un médecin omnipraticien, d'un interniste, d'un cardiologue, d'un endocrinologue ou d'un néphrologue, une consultation à l'urgence, des consultations médicales ambulatoires fréquentes sans égard à la spécialité (42 services ou plus), des consultations ambulatoires fréquentes d'un omnipraticien (22 services ou plus), des consultations ambulatoires fréquentes de spécialistes (4 services ou plus) ou des consultations fréquentes à l'urgence (4 services ou plus). Pour mieux tenir compte du contexte québécois, les seuils utilisés pour définir l'utilisation fréquente ont été basés sur les quartiles populationnels tirés d'une population avec HTA, diabète ou dyslipidémie diagnostiqués entre 2006 et 2007 au Québec (renseignements sur la recherche disponibles sur demande). Par exemple, $25 \%$ (quartile supérieur) des patients de cette population avaient reçu au moins 22 services d'un omnipraticien. Dépassés ces seuils, les patients ont donc été considérés comme grands utilisateurs de services de santé. Naessens et collab. ${ }^{34}$ ont choisi pour leur part d'utiliser un seuil de 10 consultations ou plus par année (avec un total de 20 sur 2 ans) pour identifier les grands utilisateurs de soins en première ligne. Mesurer la proportion de ces grands utilisateurs de soins de santé est important, car l'utilisation des soins et les coûts qui leur sont associés sont attribuables à une fraction relativement réduite de la population. À titre d'exemple, aux États-Unis, $5 \%$ de la population est à l'origine d'environ $50 \%$ de tous les coûts de santé ${ }^{35}$.

Enfin, pour les variables dépendantes liées aux médicaments, les proportions ont été calculées sur les personnes ayant survécu

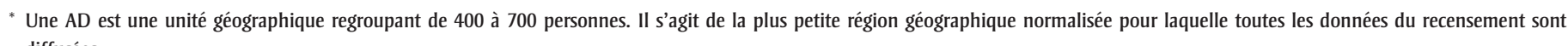
diffusées. 
au terme de la période de deux ans suivant la date de référence et admissibles au régime public d'assurance médicaments du Québec. Ces variables sont l'utilisation d'un antihypertenseur toutes causes confondues et l'utilisation d'un antihypertenseur selon sa classe (inhibiteur de l'enzyme de conversion de l'angiotensine, antagoniste des récepteurs de l'angiotensine II, diurétique, bêtabloquant, bloqueur des canaux calciques, autre). On considère qu'un patient a utilisé un médicament pour une classe spécifique s'il a présenté à la pharmacie au moins une ordonnance de cette classe dans les deux ans suivant la date de référence.

Pour déterminer le type de milieu de vie (région métropolitaine ou non métropolitaine), nous avons utilisé la classification des secteurs statistiques (CSS) développée par Statistique Canada ${ }^{36}$ dont les unités de base sont les municipalités. Chaque municipalité appartient ainsi à une région métropolitaine de recensement (RMR) (au moins 100000 habitants), à une agglomération de recensement (AR) ou une petite ville (entre 10000 et 99999 habitants) ou encore à une région rurale ou une zone d'influence métropolitaine (ZIM) forte à nulle (si la municipalité n'est pas classée ailleurs). Comme le préconise Statistique Canada ${ }^{37}$, nous avons regroupé ici les petites villes et les zones rurales dans la catégorie " régions non métropolitaines ».

Pour le niveau de défavorisation du secteur de résidence, l'INSPQ a développé un indice de défavorisation à l'aide de six indicateurs socioéconomiques ${ }^{38-42}$ calculés au niveau des AD. La composante matérielle de l'indice a été calculée en tenant compte de la proportion de personnes sans diplôme d'études secondaires, du ratio emploi/population et du revenu moyen, tandis que la composante sociale a été calculée à l'aide de la proportion de personnes vivant seules, de la proportion de personnes séparées, divorcées ou veuves et de la proportion de familles monoparentales. Les $\mathrm{AD}$ ont été classées en quintiles (chacun représentant par définition $20 \%$ de la population). Le quintile 1 (Q1) représente la population la plus favorisée et, inversement, le quintile 5 (Q5), la plus défavorisée. Ces opérations ont été effectuées séparément pour les composantes matérielle et sociale puis ont été croisées, ce qui a permis de constituer ainsi 25 classes de défavorisation du secteur de résidence $(\mathrm{Q} 1 \times \mathrm{Q} 1$ à $\mathrm{Q} 5 \times \mathrm{Q} 5)$.

\section{Analyses statistiques}

Les analyses ont été effectuées sur l'ensemble de la cohorte et ont été stratifiées par type de milieu de vie (régions métropolitaines et non métropolitaines). Pour déterminer si les différences dans les indicateurs de santé étaient statistiquement significatives entre les régions métropolitaines et non métropolitaines, nous avons utilisé le test du chi-2. Compte tenu de la taille de la cohorte à l'étude $(\mathrm{N}=276793)$, des différences minimes pourraient s'avérer statistiquement significatives. Par conséquent, nous avons également utilisé le concept de signification clinique, selon lequel une variation positive ou négative de $10 \%$ ou plus des indicateurs de santé est considérée comme cliniquement significative. Pour chaque variable dépendante à l'étude, les proportions ont été ajustées pour l'âge et le sexe. La variabilité des indicateurs de santé selon la défavorisation a été mesurée à l'aide d'un coefficient de variation $(\mathrm{CV})$, qui représente le rapport entre l'écart-type et la moyenne. Pour comparer la proportion ajustée de la classe d'intérêt (l'une des 25 classes de défavorisation matérielle et sociale) et la proportion observée dans la classe la moins défavorisée matériellement et socialement $(\mathrm{Q} 1 \times \mathrm{Q} 1)$, nous avons calculé le risque relatif (RR), qui indique le pourcentage d'augmentation ou de diminution du risque par rapport à cette classe $(\mathrm{Q} 1 \times \mathrm{Q} 1)$, et auquel nous avons associé un intervalle de confiance (IC).

\section{Résultats}

Au total, 472558 personnes de 30 ans et plus répondaient à nos critères d'inclusion pour la période considérée (du premier janvier 2006 au 31 décembre 2007) (figure 1). Parmi elles, 180328 (38,2 \%) avaient reçu un diagnostic de MCV dans les 4 ans précédant la date de référence ou présentaient un problème d'hypertension artérielle potentiellement lié à une grossesse $(n=1757)$ et ont été de ce fait exclues.
Ont été également exclues 13680 personnes dont le lieu de résidence était invalide ou manquant, ou qui résidaient dans des $\mathrm{AD}$ pour lesquelles aucune information socioéconomique n'était disponible. La cohorte finale comptait donc 276793 individus.

De ce nombre, environ $70 \%$ étaient couverts par le régime public d'assurance médicaments à la date de référence, principalement à cause de l'âge (âge moyen de 66 ans, avec $57 \%$ de personnes de 65 ans et plus) (tableau 1). Les deux tiers résidaient dans des régions métropolitaines et un tiers dans des régions non métropolitaines (12\% dans des petites villes et $20 \%$ dans des régions rurales). Cette répartition est sensiblement la même que celle de l'ensemble de la population des résidents du Québec de 30 ans et plus.

La répartition de la cohorte selon la défavorisation matérielle et sociale du secteur de résidence n'est pas uniforme, en raison d'une surreprésentation de patients dans les secteurs les plus défavorisés, ceux des quatrième $(\mathrm{Q} 4)$ et cinquième quintiles (Q5) (tableau 1). Ceci serait le signe d'une prévalence d'HTA en prévention primaire plus importante dans les secteurs défavorisés (figure 2).

Les résultats des analyses globales et des analyses selon le milieu de vie sont présentés dans le tableau 2. Bien que presque toutes les valeurs soient statistiquement différentes entre les régions métropolitaines et les régions non métropolitaines, la signification clinique de ces différences (au moins $\pm 10 \%$ ) est beaucoup moins importante, notamment pour l'utilisation de médicaments. Parmi les résultats qui témoignent d'une différence cliniquement significative, on compte la proportion de décès et d'hospitalisations toutes causes confondues ainsi que la proportion d'événements cardiovasculaires, les régions métropolitaines présentant des valeurs moins élevées que les régions non métropolitaines. De plus, les patients des régions métropolitaines consultent en plus grande proportion des spécialistes, mais consultent moins à l'urgence.

Le tableau 3 rapporte les variations observées pour chaque indicateur de santé 
FIGURE 1

Sélection de la cohorte à l'étude

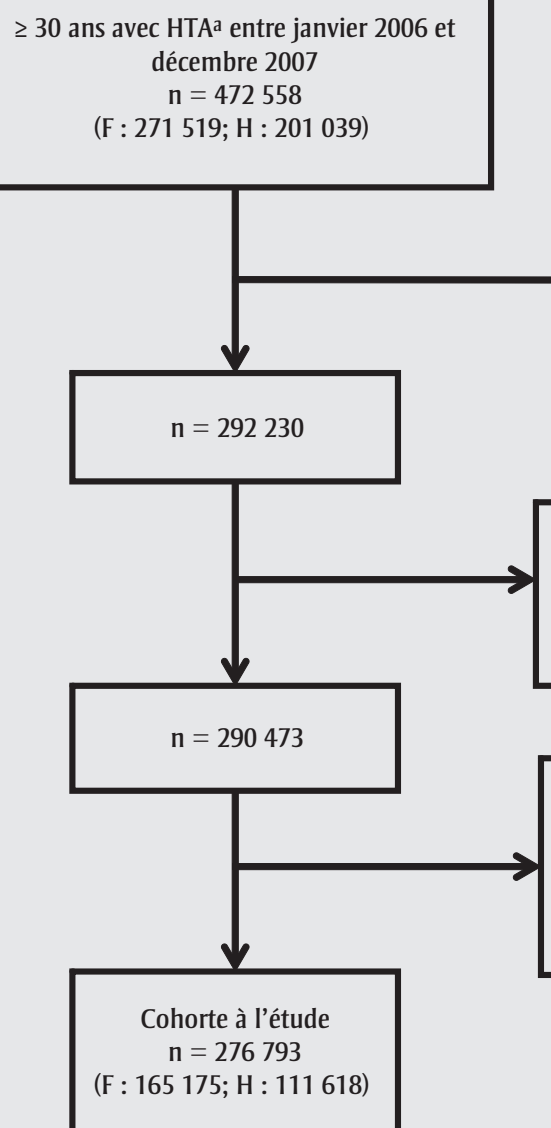

Exclusion : diagnostic de MCV dans les 4 ans précédant

la date du 1 er diagnostic HTA $n=180328(38,2 \%)$

Exclusion : diagnostic lié à une grossesse dans les 5 mois suivant la date du 1 er diagnostic HTA $\mathrm{n}=1757(0,6 \%)$

Exclusion : code de lieu de résidence erroné, manquant ou pour lequel aucune information sur la défavorisation n'est disponible n $=13680(4,7 \%)$

Abréviations : CIM, classification internationale des maladies; F, femmes; H, hommes; HTA, hypertension artérielle; MCV, maladies cardiovasculaires.

${ }^{\text {a }}$ Hospitalisés avec un diagnostic principal ou secondaire d'HTA (CIM-9 : 401 ou CIM-10 : 110) ou ayant utilisé au moins trois services médicaux en 365 jours avec un diagnostic d'HTA durant la période à l'étude.

après ajustement pour l'âge et le sexe au sein des 25 types de défavorisation des secteurs, et ce, pour l'ensemble et selon le milieu de vie (régions métropolitaines et non métropolitaines). Parmi les indicateurs présentant une importante variation selon la défavorisation, on trouve la proportion de décès, la proportion d'événements cardiovasculaires, les proportions de grands utilisateurs de soins ambulatoires chez un omnipraticien et de soins ambulatoires chez des médecins spécialistes ainsi que la proportion de grands utilisateurs de l'urgence. Peu ou pas de variation n'est percevable quant à l'utilisation de médicaments antihypertenseurs.

Comparés aux patients vivant dans des secteurs favorisés matériellement et socialement $(\mathrm{Q} 1 \times \mathrm{Q} 1)$, les patients vivant dans les secteurs les plus défavorisés matériellement et socialement (Q5 $\times$ Q5) avaient $58 \%$ plus de risque de mourir $(\mathrm{RR}=1,58$, IC à $95 \%: 1,41$ à 1,77), $46 \%$ plus de risque d'événement cardiovasculaire ( $R R=1,46$, IC à $95 \%$ : 1,29 à 1,65), $47 \%$ plus de risque d'être grands utilisateurs de l'urgence $(\mathrm{RR}=1,47$, IC à $95 \%: 1,40$ à 1,55), et $31 \%$ plus de risque d'être grands utilisateurs des services d'un omnipraticien ( $\mathrm{RR}=1,31$, IC à $95 \%: 1,25$ à 1,38) (tableau 3). En revanche, les patients vivant dans les secteurs les plus défavorisés matériellement et socialement avaient $25 \%$ moins de chances d'être de grands utilisateurs des services de médecins spécialistes $(\mathrm{RR}=0,75$, IC à $95 \%$ : 0,71 à 0,79).

La figure 2 présente les variations observées dans la prévalence d'HTA en préven- tion primaire selon la défavorisation matérielle et sociale. Cette représentation graphique permet de mieux visualiser la variation liée à la défavorisation matérielle du secteur et la variation liée à sa défavorisation sociale. On remarque un important gradient des prévalences vers les secteurs les plus défavorisés. Néanmoins, une fois ajustée pour l'âge et le sexe, la distribution selon le type de secteur est beaucoup plus uniforme. Le gradient persiste toutefois dans les régions métropolitaines.

Les figures 3 à 5 présentent les variations observées pour une sélection d'indicateurs de santé ayant présenté un CV relativement élevé (tableau 2), dans l'ensemble (figure 3) et selon le milieu de vie (figures 4 et 5). Les indicateurs ajustés pour l'âge et le sexe présentant un important gradient général des secteurs les moins défavorisés aux plus défavorisés (figure 3) sont la proportion de décès, la proportion d'événements cardiovasculaires, la proportion de grands utilisateurs des services ambulatoires (omnipraticien, urgence). Il est intéressant de noter que la relation s'inverse pour la proportion ajustée de grands utilisateurs de médecins spécialistes, les personnes vivant dans les secteurs les plus favorisés matériellement utilisant plus fréquemment les services de spécialistes. Les deux types de défavorisation semblent jouer un rôle équivalent pour certains indicateurs (proportion de décès, proportion de grands utilisateurs d'omnipraticiens) alors que pour d'autres indicateurs (proportion d'événements cardiovasculaires, proportion de grands utilisateurs de l'urgence ou de spécialistes), la défavorisation matérielle joue un rôle prédominant.

Les gradients observés dans les régions métropolitaines (figure 4) correspondent sensiblement à ceux observés pour l'ensemble (figure 3), probablement en raison du fait que ces régions représentent les deux tiers de la cohorte à l'étude. Les analyses stratifiées selon le milieu de vie révèlent des variations plus importantes dans les régions non métropolitaines (figure 5) que dans les régions métropolitaines (figure 4). Ces variations ne sont pas surprenantes étant donné les échantillons plus petits correspondant à ces régions, ce qui cause une variance plus grande dans l'estimation des proportions. 
TABLEAU 1

Caractéristiques de la cohorte à l'étude $(n=276$ 793) selon le type de milieu

\begin{tabular}{|c|c|c|c|c|}
\hline \multirow[t]{2}{*}{ Caractéristiques } & \multirow[t]{2}{*}{ Total } & \multirow{2}{*}{$\begin{array}{c}\text { Régions } \\
\text { métropolitaines }^{\mathrm{a}}\end{array}$} & \multicolumn{2}{|c|}{ Régions non métropolitaines } \\
\hline & & & Petites villes ${ }^{b}$ & Régions rurales \\
\hline Cohorte à l'étude, n (\%) & $276793(100)$ & $188107(68,0)$ & $33127(12,0)$ & $55559(20,0)$ \\
\hline Âgé moyen en années (écart-type) & $66,3(12,7)$ & $66,0(12,7)$ & $67,0(12,5)$ & $66,7(12,4)$ \\
\hline \multicolumn{5}{|l|}{ Groupe d'âge, n (\%) } \\
\hline Moins de 65 ans & $117844(42,6)$ & $81793(43,5)$ & $13231(39,9)$ & $22820(41,1)$ \\
\hline 65 ans et plus & $158949(57,4)$ & $106314(56,5)$ & $19896(60,1)$ & $32739(58,9)$ \\
\hline \multicolumn{5}{|l|}{ Sexe, n (\%) } \\
\hline Femme & $165175(59,7)$ & $112663(59,9)$ & $20299(61,3)$ & $32213(58,0)$ \\
\hline Homme & $111618(40,3)$ & $75444(40,1)$ & $12828(38,7)$ & $23346(42,0)$ \\
\hline \multicolumn{5}{|c|}{ Quintile de défavorisation matérielle, n (\%) } \\
\hline $\mathrm{Q} 1^{\mathrm{c}}$ & $48585(17,6)$ & $44908(23,9)$ & $2097(6,3)$ & $1580(2,8)$ \\
\hline Q2 & $53203(19,2)$ & $42833(22,8)$ & $5887(17,8)$ & $4483(8,1)$ \\
\hline Q3 & $57577(20,8)$ & $39721(21,1)$ & $8661(26,1)$ & $9195(29,2)$ \\
\hline Q4 & $59302(21,4)$ & $34506(18,3)$ & $8562(25,8)$ & $16234(29,2)$ \\
\hline$Q 5^{\mathrm{d}}$ & $58126(21,0)$ & $26139(13,9)$ & $7920(23,9)$ & $24067(43,3)$ \\
\hline \multicolumn{5}{|c|}{ Quintile de défavorisation sociale, n (\%) } \\
\hline $\mathrm{Q} 1^{\mathrm{c}}$ & $47248(17,1)$ & $29772(15,8)$ & $4438(13,4)$ & $13038(23,5)$ \\
\hline Q2 & $50861(18,4)$ & $28227(15,0)$ & $5403(16,3)$ & $17231(31,0)$ \\
\hline Q3 & $55106(19,9)$ & $33892(18,0)$ & $6532(19,7)$ & $14682(26,4)$ \\
\hline Q4 & $60820(22,0)$ & $45199(24,0)$ & $7417(22,4)$ & $8204(14,8)$ \\
\hline$Q 5^{\mathrm{d}}$ & $62758(22,7)$ & $51017(27,1)$ & $9337(28,2)$ & $2404(4,3)$ \\
\hline \multicolumn{5}{|c|}{ Croisement des quintiles de défavorisation matérielle et sociale, n (\%) } \\
\hline $\mathrm{Q} 1 \times \mathrm{Q} 1$ & $8900(3,2)$ & $8368(4,4)$ & $338(1,0)$ & $194(0,4)$ \\
\hline $\mathrm{Q} 1 \times \mathrm{Q} 2$ & $7340(2,6)$ & $6629(3,5)$ & $439(1,3)$ & $272(0,5)$ \\
\hline Q1 × Q3 & $9143(3,3)$ & $8020(4,3)$ & $494(1,5)$ & $629(1,1)$ \\
\hline $\mathrm{Q} 1 \times \mathrm{Q} 4$ & $11137(4,0)$ & $10488(5,6)$ & $321(1,0)$ & $328(0,6)$ \\
\hline Q1 × Q5 & $12065(4,4)$ & $11403(6,1)$ & $505(1,5)$ & $157(0,3)$ \\
\hline $\mathrm{Q} 2 \times \mathrm{Q} 1$ & $8649(3,1)$ & $7177(3,8)$ & $813(2,4)$ & $659(1,2)$ \\
\hline $\mathrm{Q} 2 \times \mathrm{Q} 2$ & $9693(3,5)$ & $7313(3,9)$ & $1158(3,5)$ & $1222(2,2)$ \\
\hline $\mathrm{Q} 2 \times \mathrm{Q} 3$ & $10745(3,9)$ & $7962(4,2)$ & $1555(4,7)$ & $1228(2,2)$ \\
\hline $\mathrm{Q} 2 \times \mathrm{Q} 4$ & $12357(4,5)$ & $9924(5,3)$ & $1220(3,7)$ & $1213(2,2)$ \\
\hline $\mathrm{Q} 2 \times \mathrm{Q} 5$ & $11759(4,2)$ & $10457(5,6)$ & $1141(3,4)$ & $161(0,3)$ \\
\hline $\mathrm{Q} 3 \times \mathrm{Q} 1$ & $9375(3,4)$ & $6390(3,4)$ & $1327(4,0)$ & $1658(3,0)$ \\
\hline $\mathrm{Q} 3 \times \mathrm{Q} 2$ & $10888(3,9)$ & $6186(3,3)$ & $1755(5,3)$ & $2947(5,3)$ \\
\hline $\mathrm{Q} 3 \times \mathrm{Q} 3$ & $12172(4,4)$ & $7838(4,2)$ & $1683(5,1)$ & $2651(4,8)$ \\
\hline $\mathrm{Q} 3 \times \mathrm{Q} 4$ & $12797(4,6)$ & $9165(4,9)$ & $2081(6,3)$ & $1551(2,8)$ \\
\hline $\mathrm{Q} 3 \times \mathrm{Q} 5$ & $12345(4,5)$ & $10142(5,4)$ & $1815(3,6)$ & $388(0,7)$ \\
\hline $\mathrm{Q} 4 \times \mathrm{Q} 1$ & $9636(3,5)$ & $5087(2,7)$ & $1183(3,6)$ & $3366(6,1)$ \\
\hline $\mathrm{Q} 4 \times \mathrm{Q} 2$ & $10445(3,8)$ & $4564(2,4)$ & $1132(3,4)$ & $4749(8,6)$ \\
\hline $\mathrm{Q} 4 \times \mathrm{Q} 3$ & $12305(4,4)$ & $5970(3,2)$ & $1727(5,2)$ & $4608(8,3)$ \\
\hline $\mathrm{Q} 4 \times \mathrm{Q} 4$ & $13429(4,8)$ & $8347(4,4)$ & $2401(7,2)$ & $2681(4,8)$ \\
\hline $\mathrm{Q} 4 \times \mathrm{Q} 5$ & $13487(4,9)$ & $10538(5,6)$ & $2119(6,4)$ & $830(1,5)$ \\
\hline $\mathrm{Q} 5 \times \mathrm{Q} 1$ & $10688(3,9)$ & $2750(1,5)$ & $777(2,4)$ & $7161(12,9)$ \\
\hline $\mathrm{Q} 5 \times \mathrm{Q} 2$ & $12495(4,5)$ & $3535(1,9)$ & $919(2,8)$ & $8041(14,5)$ \\
\hline $\mathrm{Q} 5 \times \mathrm{Q} 3$ & $10741(3,9)$ & $4102(2,2)$ & $1073(3,2)$ & $5566(10,0)$ \\
\hline
\end{tabular}

Suite page suivante
Dans l'ensemble, la plupart des variables étudiées présentent néanmoins des résultats allant dans le même sens, sauf peutêtre pour la mortalité et les événements cardiovasculaires, où une plus grande instabilité est observée dans les estimations.

\section{Analyse}

Cette étude montre d'importantes variations pour plusieurs indicateurs de santé selon la défavorisation matérielle et sociale du secteur. Même après ajustement pour l'âge et le sexe, le risque de décès était plus élevé de $58 \%$, le risque d'événement cardiovasculaire de $46 \%$, le risque d'hospitalisation (toutes causes confondues) de $18 \%$ et la prévalence de $14 \%$ pour les personnes hypertendues vivant dans les secteurs les plus défavorisés $(\mathrm{Q} 5 \times \mathrm{Q} 5)$ par rapport à celles vivant dans les secteurs les moins défavorisés $(\mathrm{Q} 1 \times \mathrm{Q} 1)$ matériellement et socialement. De plus, la proportion ajustée de grands utilisateurs de services de santé de première ligne était beaucoup plus importante dans les secteurs les plus défavorisés, avec $47 \%$ plus de grands utilisateurs de l'urgence et $31 \%$ plus de grands utilisateurs des services d'omnipraticiens. D’autres études ont déjà démontré que les individus ayant un statut socioéconomique plus faible utilisent davantage les services médicaux ambulatoires, dont les services à l'urgence ${ }^{43}$. Il est intéressant de noter que, dans notre étude, non seulement les patients des secteurs les plus défavorisés consultaient plus à l'urgence, ce qu'a déjà relevé une autre étude canadienne ${ }^{44}$, mais consultaient également davantage les omnipraticiens. On a remarqué aussi une plus grande proportion de grands utilisateurs de services spécialisés chez les résidents des secteurs les mieux nantis. Ces variations étaient également présentes dans les analyses stratifiées selon les régions métropolitaines et non métropolitaines. Quant à l'absence de variation (ou la faible variation) percevable dans l'utilisation de médicaments antihypertenseurs, elle pourrait témoigner de l'impact favorable de la politique provinciale d'accès universel aux médicaments. 
TABLEAU 1 (Suite)

Caractéristiques de la cohorte à l'étude $(n=276$ 793) selon le type de milieu

\begin{tabular}{|c|c|c|c|c|}
\hline \multirow[t]{2}{*}{ Caractéristiques } & \multirow[t]{2}{*}{ Total } & \multirow{2}{*}{$\begin{array}{c}\text { Régions } \\
\text { métropolitaines }^{\mathrm{a}}\end{array}$} & \multicolumn{2}{|c|}{ Régions non métropolitaines } \\
\hline & & & Petites villes $^{\mathbf{b}}$ & Régions rurales \\
\hline $\mathrm{Q} 5 \times \mathrm{Q} 4$ & $11110(4,0)$ & $7275(3,9)$ & $1394(4,2)$ & $2431(4,4)$ \\
\hline Q5 $\times$ Q5 & $13102(4,7)$ & $8477(4,5)$ & $3757(11,3)$ & $868(1,6)$ \\
\hline
\end{tabular}

a Correspond à une région métropolitaine de recensement (au moins 100000 habitants) ${ }^{36}$.

b Petite ville (entre 10000 et 99999 habitants) ou agglomération de recensement ou encore région rurale ou une zone d'influence métropolitaine forte à nulle, regroupées ici dans une même catégorie, tel que préconisé par Statistique Canada ${ }^{37}$.

c Le plus favorisé.

d Le plus défavorisé.
En résumé, les patients hypertendus sans antécédents de MCV vivant dans des secteurs défavorisés matériellement et socialement subissent, dans une plus grande proportion, des conséquences plus graves que ceux vivant dans des secteurs favorisés, et ce, malgré le fait qu'ils bénéficient d'un traitement médicamenteux équivalent.

Dans notre étude, la prévalence d'HTA en prévention primaire des MCV est estimée

FIGURE 2

Inégalités dans la prévalence d'HTA en prévention primaire des MCV selon la défavorisation du secteur et le type de milieu (régions métropolitaines ou non) : risques relatifs
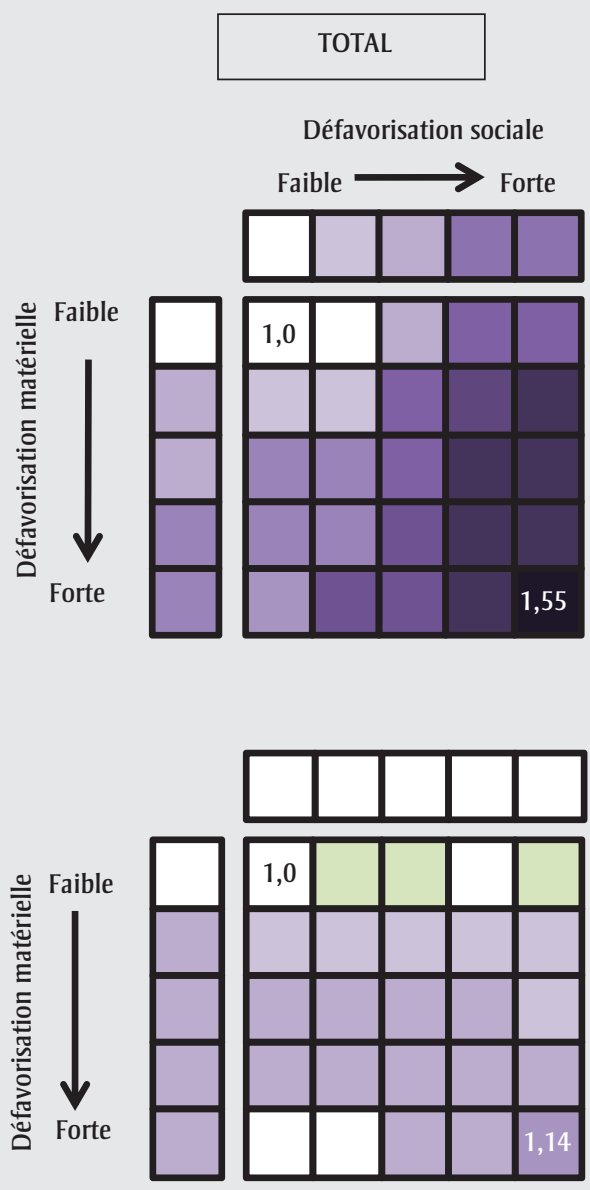

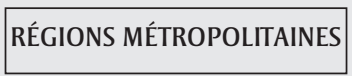

Défavorisation sociale
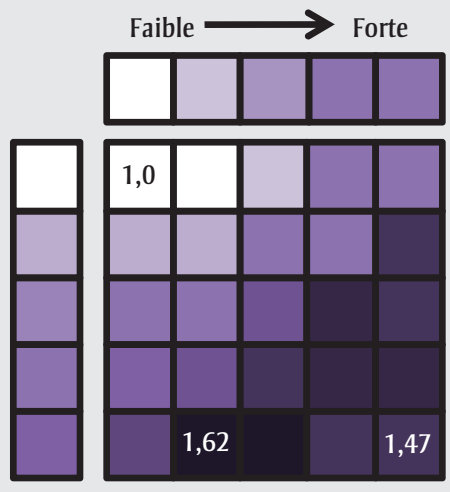

Prévalence HTA brute
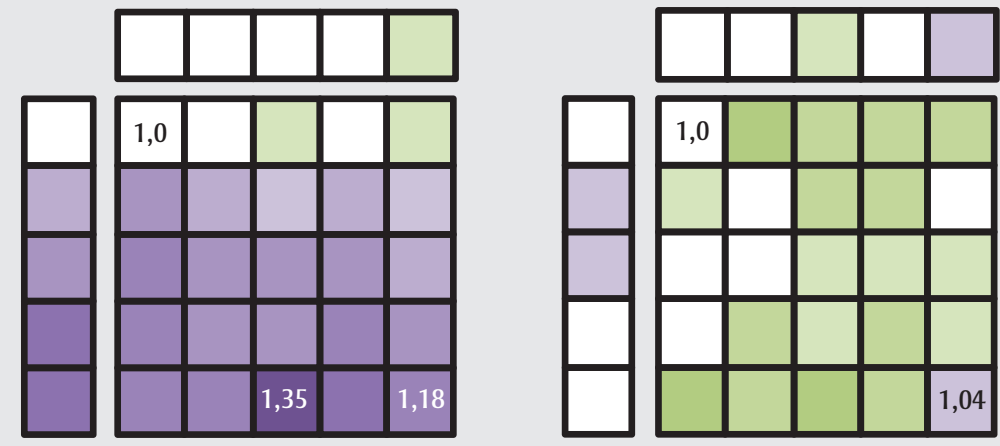

Prévalence HTA ajustée pour l'âge et le sexe

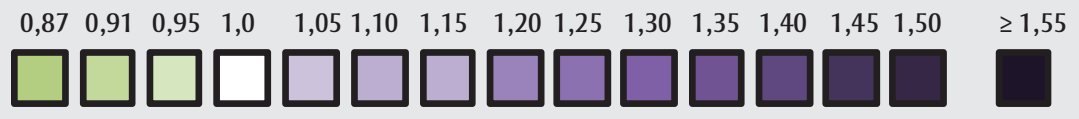

RR

Abréviations : HTA, hypertension artérielle; MCV, maladie cardiovasculaire; RR, risque relatif.

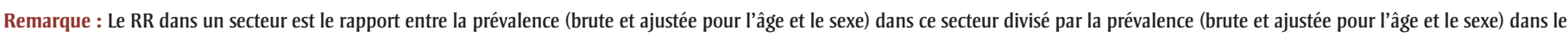
secteur le plus favorisé matériellement et socialement. 
TABLEAU 2

Description des indicateurs de santé chez les patients (âgés de 30 ans et plus) hypertendus en prévention primaire des MCV, globalement et selon le type de milieu (régions métropolitaines ou non), Québec, janvier 2006 à décembre 2007

\begin{tabular}{|c|c|c|c|c|c|c|c|c|c|c|c|}
\hline \multirow[t]{2}{*}{ Indicateurs de santéa } & \multicolumn{3}{|c|}{ Total } & \multicolumn{3}{|c|}{ Régions métropolitaines ${ }^{b}$} & \multicolumn{3}{|c|}{ Régions non métropolitaines ${ }^{c}$} & \multirow[t]{2}{*}{ Valeur $p^{d}$} & \multirow{2}{*}{$\begin{array}{c}\text { Variation } \\
(\text { en \% })^{\mathrm{e}}\end{array}$} \\
\hline & $\mathbf{n}$ & $\mathbf{N}$ & $\%$ & $\mathbf{n}$ & $\mathbf{N}$ & $\%$ & $\mathbf{n}$ & $\mathbf{N}$ & $\%$ & & \\
\hline $\begin{array}{l}\text { Prévalence HTA en prévention } \\
\text { primaire des MCV }\end{array}$ & 276793 & 4697515 & 5,9 & 188107 & 3143625 & 6,0 & 88686 & 1553415 & 5,7 & $<0,001$ & $-5,0$ \\
\hline Décès & 15104 & 276793 & 5,5 & 9677 & 188107 & 5,1 & 5427 & 88686 & 6,1 & $<0,001$ & 19,6 \\
\hline Événement cardiovasculaire & 14050 & 276793 & 5,1 & 8665 & 188107 & 4,6 & 5385 & 88686 & 6,1 & $<0,001$ & 32,6 \\
\hline $\begin{array}{l}\text { Hospitalisation toutes causes } \\
\text { confondues }\end{array}$ & 87395 & 261689 & 33,4 & 56387 & 178430 & 31,6 & 31008 & 83259 & 37,2 & $<\mathbf{0 , 0 0 1}$ & 17,7 \\
\hline \multicolumn{12}{|l|}{ Nombre de consultations } \\
\hline Omnipraticien $(\geq 1)$ & 256657 & 261689 & 98,1 & 174853 & 178430 & 98,0 & 81804 & 83259 & 98,3 & $<0,001$ & 0,3 \\
\hline Spécialiste $(\geq 1)$ & 103207 & 261689 & 39,4 & 73809 & 178430 & 41,4 & 29398 & 83259 & 35,3 & $<0,001$ & $-14,7$ \\
\hline Cardiologue $(\geq 1)$ & 64140 & 261689 & 24,5 & 47873 & 178430 & 26,8 & 16267 & 83259 & 19,5 & $<0,001$ & $-27,2$ \\
\hline Interniste $(\geq 1)$ & 42269 & 261689 & 16,2 & 27766 & 178430 & 15,6 & 14503 & 83259 & 17,4 & $<0,001$ & 11,5 \\
\hline \multicolumn{12}{|l|}{ Grands utilisateurs } \\
\hline Omnipraticiens $(\geq 22)$ & 66601 & 261689 & 25,4 & 43157 & 178430 & 24,2 & 23444 & 83259 & 28,2 & $<0,001$ & 16,5 \\
\hline Spécialistes $(\geq 4)$ & 48899 & 261689 & 18,7 & 36713 & 178430 & 20,6 & 12186 & 83259 & 14,6 & $<0,001$ & $-29,1$ \\
\hline Urgence $(\geq 4)$ & 63992 & 261689 & 24,5 & 41131 & 178430 & 23,1 & 22861 & 83259 & 27,5 & $<0,001$ & 19,0 \\
\hline Antihypertenseur & 175204 & 183156 & 95,7 & 115376 & 120833 & 95,5 & 59828 & 62323 & 96,0 & $<0,001$ & 0,5 \\
\hline IECA/ARA & 136061 & 183156 & 74,3 & 89617 & 120833 & 74,2 & 46444 & 62323 & 74,5 & 0,0991 & 0,4 \\
\hline Diurétique & 117660 & 183156 & 64,2 & 78632 & 120833 & 65,1 & 39028 & 62323 & 62,6 & $<0,001$ & $-3,8$ \\
\hline Bloqueur des canaux calciques & 85305 & 183156 & 46,6 & 55288 & 120833 & 45,8 & 30017 & 62323 & 48,2 & $<0,001$ & 5,2 \\
\hline Bêtabloquant & 63678 & 183156 & 34,8 & 41139 & 120833 & 34,0 & 22539 & 62323 & 36,2 & $<0,001$ & 6,5 \\
\hline
\end{tabular}

Abréviations : HTA, hypertension artérielle; IECA/ARA, inhibiteur de l'enzyme de conversion de l'angiotensine ou antagoniste des récepteurs de l'angiotensine II; MCV, maladie cardiovasculaire.

Remarque : Les indicateurs en gras indiquent un pourcentage de variation de $10 \%$ ou plus entre les valeurs des régions métropolitaines et celles des régions non métropolitaines.

${ }^{a}$ Calculé dans les 2 ans suivant la date de référence.

${ }^{\mathrm{b}}$ Correspond à une région métropolitaine de recensement (au moins 100000 habitants).

c Petite ville (entre 10000 et 99999 habitants) ou région rurale.

d Test du chi-2 pour la différence de proportion entre les régions métropolitaines et non métropolitaines.

e Pourcentage de variation entre le taux obtenu pour les régions non métropolitaines et celui obtenu pour les régions métropolitaines.

à 5,9\% sur les 2 ans étudiés (2006-2007). Notre estimé est beaucoup plus faible que la prévalence d'HTA calculée par l'Institut national de la santé publique du Québec $(\text { INSPQ })^{10}$ pour 2006-2007 (20,3\%) ou que la prévalence estimée par Lix et collab. $^{32}$. pour 2002-2003 (10,0 \%). Toutefois, il s'agit de populations à l'étude différentes : nous avons inclus seulement les patients hypertendus sans antécédents de $\mathrm{MCV}$, ce qui correspond à une réduction de près de $38 \%$ de leur nombre (figure 1). De plus, les codes diagnostics utilisés dans notre étude pour l'identification des cas d'HTA ont été restreints à un seul code (CIM-9 : 401 et CIM-10 : I10), comme l'ont fait Lix et collab. ${ }^{32}$, alors que
l'INSPQ a élargi les codes d'HTA à ceux reliés à d'autres pathologies (cardiopathie, néphropathie, cardionéphropathie due à l'hypertension artérielle et hypertension secondaire, CIM-9 : 402-405 et CIM-10 : I11-I13 et I15). Enfin, notre algorithme pour identifier les cas d'HTA (3 diagnostics en 1 an ou une hospitalisation) était plus spécifique que ceux utilisés par l'INSPQ (2 diagnostics en 2 ans ou 1 hospitalisation) et par Lix et collab. ${ }^{32}$ (2 diagnostics en 1 an ou 1 hospitalisation).

Il est aussi intéressant de souligner que la cohorte à l'étude était composée de $60 \%$ de femmes. Diverses raisons peuvent expliquer ce phénomène. Tout d'abord, les femmes sont de plus grandes utilisatrices des services de santé que les hommes $^{45-47}$, et sont donc plus susceptibles que les hommes de recevoir un diagnostic d'HTA et d'être détectées par notre algorithme de sélection. De plus, nous avons exclu tous les patients ayant déjà reçu un diagnostic de maladies cardiovasculaires, or ce sont des maladies plus fréquentes chez les hommes que chez les femmes ${ }^{48-49}$.

Le gradient observé dans les taux de prévalence d'HTA en prévention primaire des MCV selon le niveau de défavorisation du secteur de résidence est très clair et reflète en bonne partie une différence réelle 


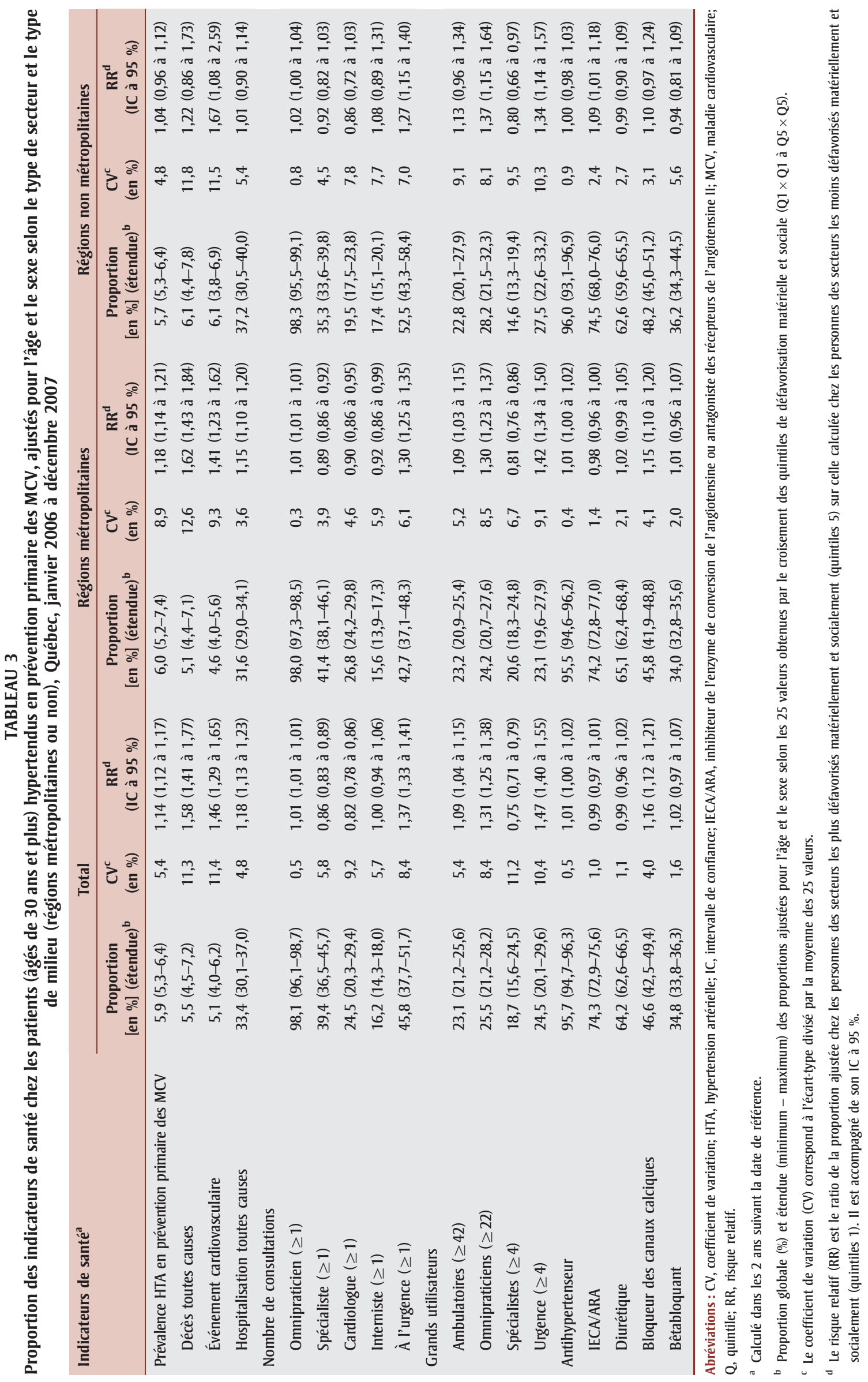


FIGURE 3

Inégalités de santé des patients souffrant d'hypertension, selon la défavorisation du secteur : risques relatifs

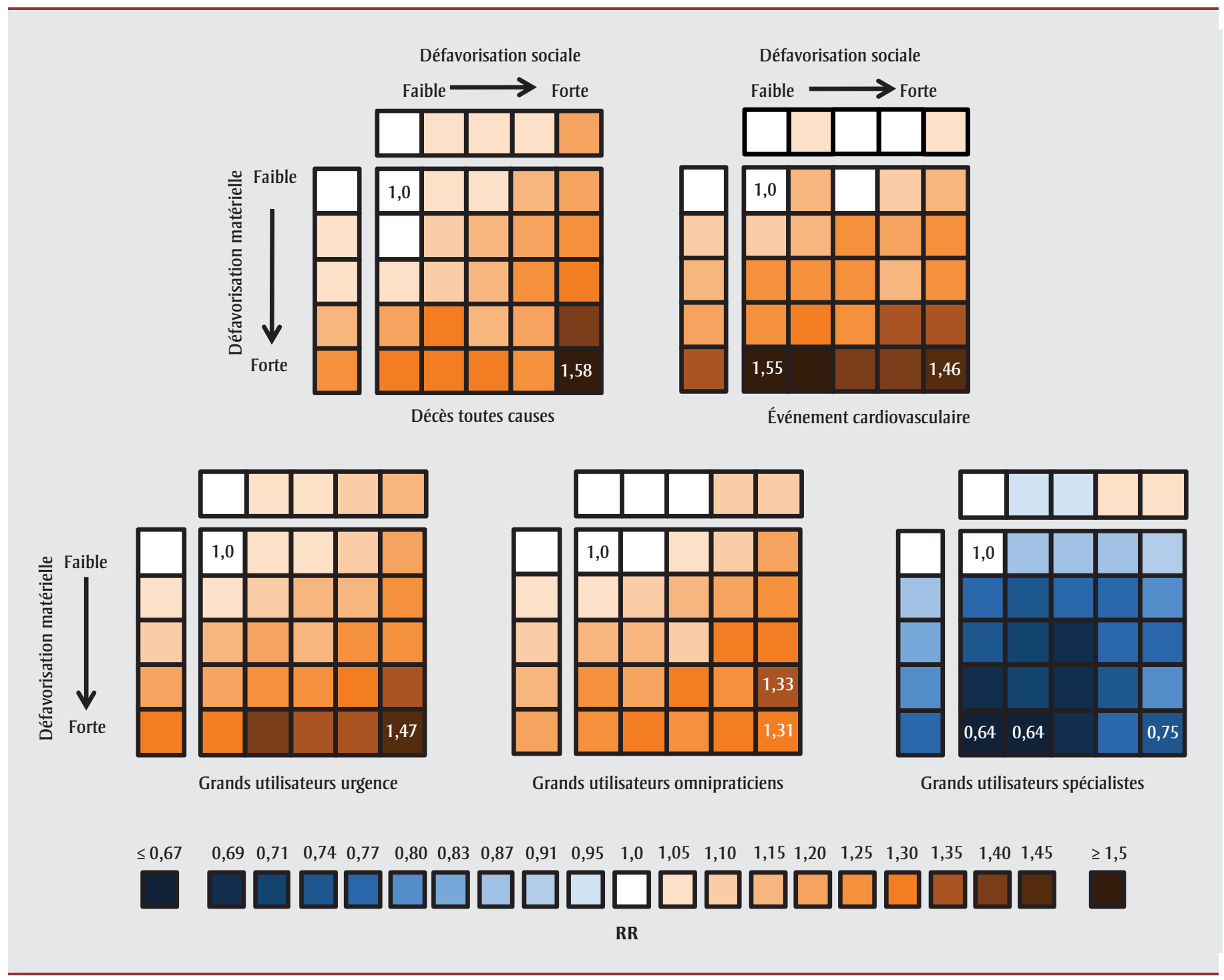

Abréviation : RR, risque relatif.

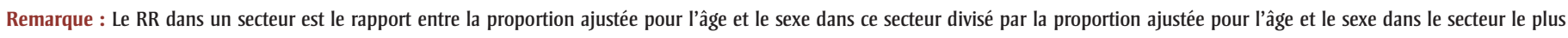
favorisé matériellement et socialement.

dans la répartition de l'âge et du sexe de ces secteurs (figure 2), comme l'illustre une diminution notable du gradient entre les résultats ajustés et non ajustés. Toutefois, nos résultats concernant la prévalence diffèrent de ceux publiés par l'INSPQ, lesquels ont fait état d'un gradient dans les taux d'incidence d'HTA des moins défavorisés vers les plus défavorisés matériellement chez les femmes seulement, avec un gradient inversé pour la défavorisation sociale chez les deux sexes $^{50}$.

Le lien entre la défavorisation et certains indicateurs de santé a fait l'objet de nombreuses études. Parmi les indicateurs de santé liés à l'hypertension artérielle spécifiquement, on compte l'incidence ${ }^{10}$, la prévalence ${ }^{51-53}$, les traitements ${ }^{54}$ et l'adéquation des soins liés à l'HTA ${ }^{55}$. L'étude de Hammouche et collab. $^{55}$ a prouvé que des participants hypertendus résidant dans des secteurs défavorisés du Royaume-Uni recevaient au moins d'aussi bons, voire de meilleurs soins, que ceux résidant dans des secteurs favorisés. L'absence de lien entre la défavorisation et l'utilisation de médicaments antihypertenseurs dans notre étude va dans le même sens que ce que Hammouche et collab. ${ }^{55}$ ont avancé mais dans le sens opposé des résultats de l'étude de Pears et collab. ${ }^{54}$ en Écosse. Cette absence de corrélation entre l'utilisation de certains médicaments et la défavorisation a aussi été observée pour une cohorte de patients souffrant de schizophrénie qui prenait des médicaments antipsychotiques ${ }^{56}$, ce qui pourrait témoigner de l'efficacité de la politique provinciale d'accès universel aux médicaments.

\section{Forces et limites}

La plus grande force de notre étude réside dans le fait que nous avons analysé l'ensemble de la population du Québec ayant reçu un diagnostic d'HTA mais sans 
FIGURE 4

Inégalités de santé des patients souffrant d’hypertension, selon la défavorisation du secteur dans les régions métropolitaines : risques relatifs
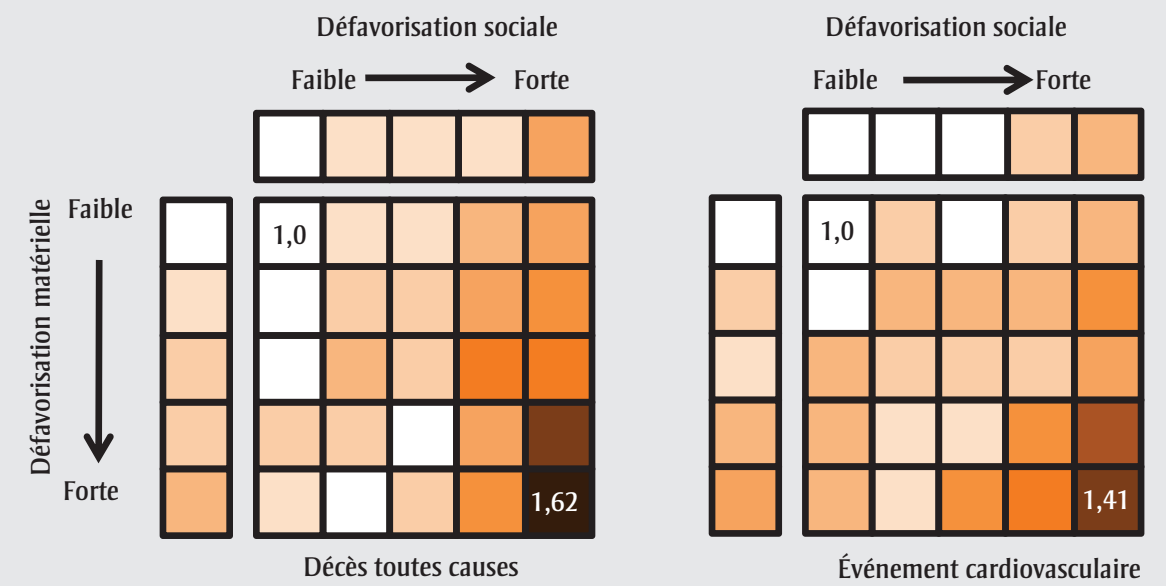

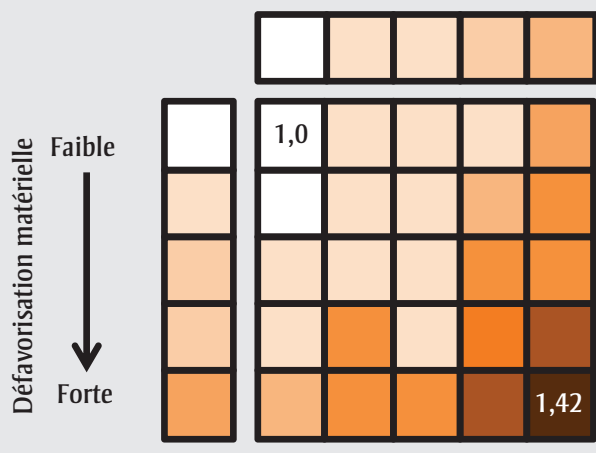

Grands utilisateurs urgence
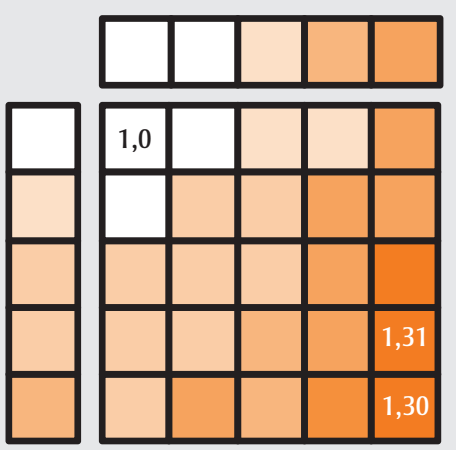

Grands utilisateurs omnipraticiens

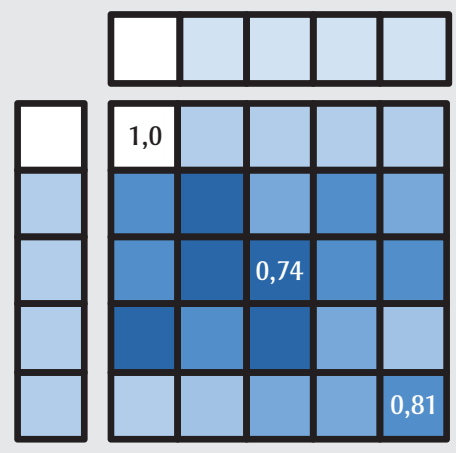

Grands utilisateurs spécialistes

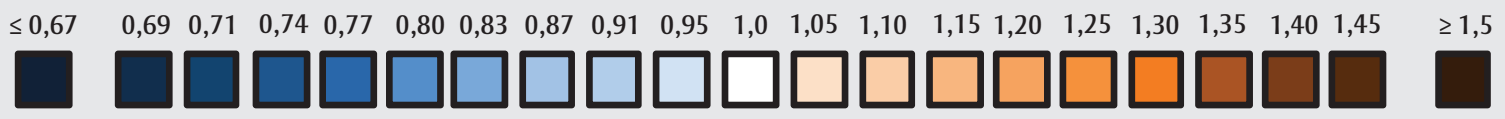

RR

Abréviation : RR, risque relatif.

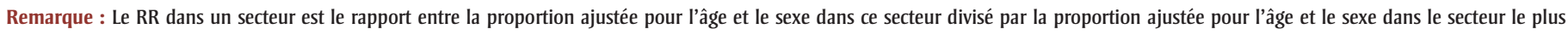
favorisé matériellement et socialement.

diagnostic connu de maladies cardiovasculaires. De plus, nous avons comparé un large éventail d'indicateurs de santé selon 25 types de secteurs, allant des plus favorisés matériellement et socialement aux plus défavorisés. Cette étude comporte néanmoins plusieurs limites. Premièrement, comme nous l'avons mentionné, l'algorithme utilisé ici est très spécifique mais peu sensible, ce qui a pour effet de sous-estimer la prévalence réelle. Nous en sommes tout à fait conscients. Toutefois, nous croyons que ce biais d'inclusion rend les analyses plus robustes concernant les autres indicateurs de santé, parce que notre cohorte comporte très peu de faux positifs. De plus, un nombre important de personnes hypertendues ne sont pas diagnostiquées ${ }^{11}$ et ne sont donc pas prises en compte dans notre cohorte, ce qui est également le cas des patients ayant consulté uniquement des médecins exerçant à tarif horaire, par exemple dans un Centre local de services communautaires (CLSC). En 2006-2007, $12 \%$ des médecins omnipraticiens pratiquaient en $\mathrm{CLSC}^{57}$.

Deuxièmement, puisque les résultats portant sur les médicaments ne concernent que $70 \%$ de notre cohorte à l'étude (183 156 patients sur 276793 étaient couverts par le régime public d'assurance médicaments), les résultats ne devraient pas être généralisés à l'ensemble de la population souffrant d'HTA (biais de sélection). De plus, les personnes âgées de moins de 65 ans défavorisées sur le plan socioéconomique sont surreprésentées dans cette sous-population. En effet, pour cette catégorie d'âge, la RAMQ couvre tous les bénéficiaires de l'aide sociale ainsi que toutes les personnes non couvertes par un régime privé d'assurance médicament. 
FIGURE 5

Inégalités de santé des patients souffrant d'hypertension, selon la défavorisation du secteur dans les régions non métropolitaines : risques relatifs

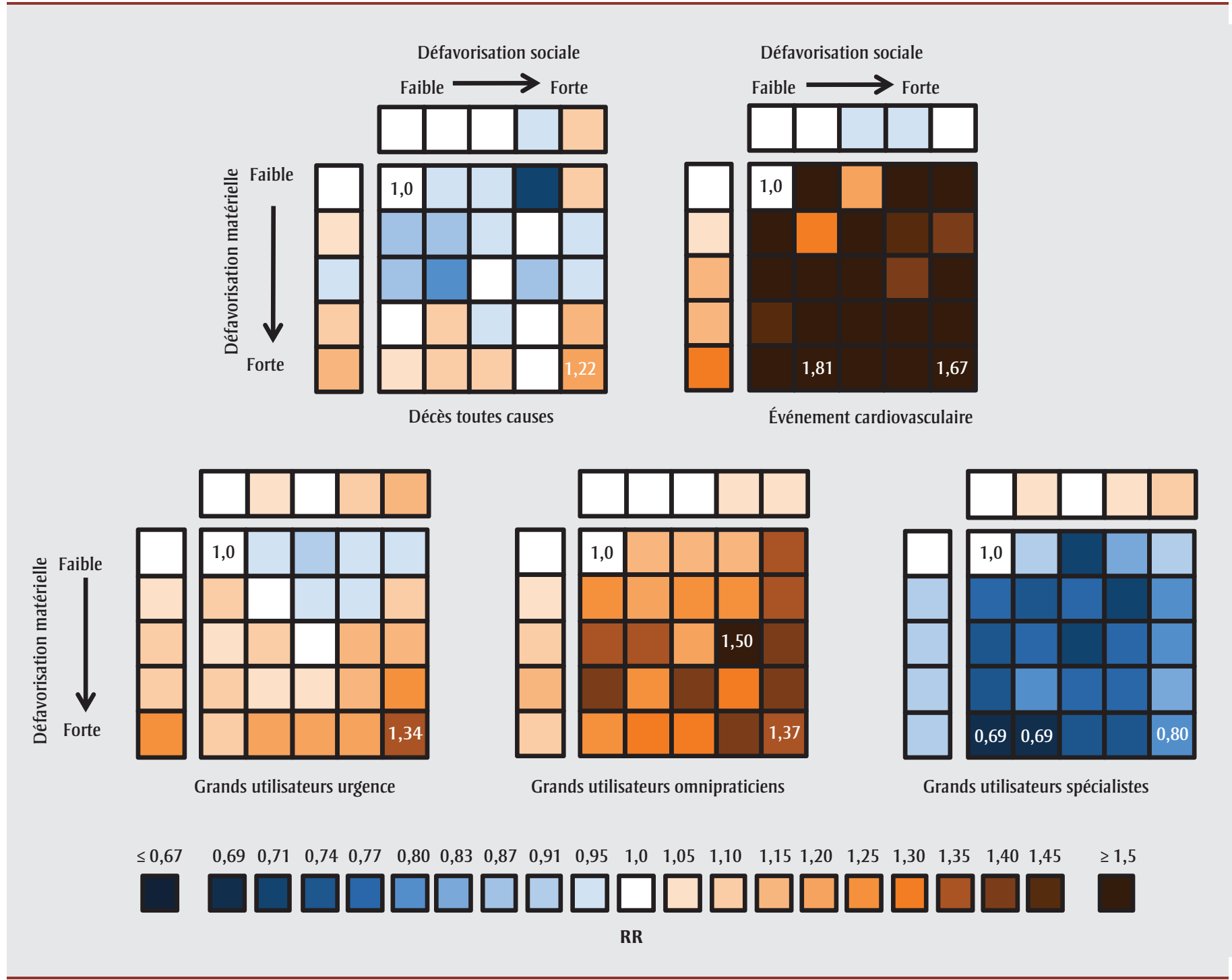

Abréviation : RR, risque relatif.

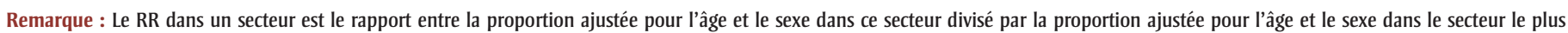
favorisé matériellement et socialement.

\section{Conclusion}

Cette étude démontre l'existence d'importantes variations pour plusieurs indicateurs de santé chez des patients hypertendus en prévention primaire des MCV en fonction de la défavorisation matérielle et sociale de leur secteur de résidence. Certains des indicateurs, comme les décès toutes causes confondues et l'incidence d'événements cardiovasculaires, peuvent conduire à une augmentation du risque allant jusqu'à $58 \%$ dans les secteurs les plus défavorisés par rapport aux moins défavorisés, et ce, malgré le fait que la variation quant à l'utilisation de médicaments antihypertenseurs soit faible ou nulle et que les patients des secteurs les plus défavorisés ne semblent pas être moins bien suivis en soins de première ligne. Dans un contexte où le fardeau des maladies chroniques est en augmentation, de telles inégalités de santé ont des répercussions majeures en termes de santé publique. Cette étude montre, encore une fois, l'importance de prendre en compte le statut socioéconomique lors de la planification d'interventions visant à prévenir les MCV et leurs conséquences. Une meilleure compréhension des processus qui sous-tendent les inégalités sociales de santé en lien avec les secteurs de résidence constitue un domaine de recherche essentiel pour la santé publique.

\section{Remerciements}

Ce projet a été financé par les Instituts de recherche en santé du Canada (IRSC). Alain Vanasse reçoit des fonds du Département de médecine de famille et de médecine d'urgence de l'Université de Sherbrooke, du Centre de recherche CHUS et du Fonds de recherche du Québec Santé (FRQS). 


\section{Références}

1. Ezzati M, Lopez AD, Rodgers A et collab. Selected major risk factors and global and regional burden of disease. Lancet. 2002; 360:1347-60.

2. Lim SS, Vos T, Flaxman AD et collab. A comparative risk assessment of burden of disease and injury attributable to 67 risk factors and risk factor clusters in 21 regions, 1990-2010 : a systematic analysis for the global burden of disease study 2010 . Lancet. 2012;380:2224-60.

3. Cloutier L, Morris D, Bruneau J, McLean D. World Health Organization celebrates World Health Day, April 7, 2013 - focusing on hypertension. Canadian Journal of Cardiovascular Nursing. 2013;23(2):9-11.

4. Kearney PM, Whelton M, Reynolds K, Muntner P, Whelton PK, He J. Global burden of hypertension : analysis of worldwide data. Lancet. 2005;365:217-23.

5. Danaei G, Finucane MM, Lin JK et collab. National, regional, and global trends in systolic blood pressure since 1980 : systematic analysis of health examination surveys and epidemiological studies with 786 country-years and 5.4 million participants. Lancet. 2011;377(9765):568-77.

6. Leenen FH, Dumais J, McInnis $\mathrm{NH}$ et collab. Results of the Ontario survey on the prevalence and control of hypertension. CMAJ. 2008;178(11):1441-9.

7. Statistique Canada. Enquête canadienne sur les mesures de la santé : la tension artérielle des adultes [Internet]. Ottawa (Ont.) : Statistique Canada; 17 février 2010 [consultation le 7 juillet 2014]. Consultable en ligne à : http://www.statcan.gc.ca/daily-quotidien /100217/dq100217b-fra.htm

8. Joffres M, Falaschetti E, Gillespie C et collab. Hypertension prevalence, awareness, treatment and control in national surveys from England, the USA and Canada, and correlation with stroke and ischaemic heart disease mortality : a cross-sectional study. BMJ Open. 2013;3(8):e003423.
9. Blais C, Rochette L. Surveillance de l'hypertension au Québec : incidence, prévalence et mortalité. Québec (Qc) : Institut national de sante publique du Québec; 2011. PDF (757 Ko) téléchargeable à partir du lien : http:// www.inspq.qc.ca/pdf/publications/1059_ HypertensionArterielle.pdf

10. Robitaille C, Dai S, Waters C et collab. Diagnosed hypertension in Canada : incidence, prevalence and associated mortality. CMAJ. 2012;184(1):E49-56.

11. Chow CK, Teo KK, Rangarajan S et collab. Prevalence, awareness, treatment, and control of hypertension in rural and urban communities in high-, middle-, and lowincome countries. JAMA. 2013;310(9):95968.

12. Organisation mondiale de la santé (OMS). Health impact assessment (HIA): the determinants of health [Internet]. Genève : OMS [consultation le 7 juillet 2014]. Consultable en ligne à la page : http://www.who.int /hia/evidence/doh/en/

13. Raphael D (dir.). Social determinants of health : Canadian perspectives. Toronto (Ont.) : Canadian Scholars’ Press; 2004.

14. Shah CP. Public health and preventive medicine in Canada ( $5^{\mathrm{e}}$ éd.). Toronto : Elsevier Canada; 2003.

15. Starfield B. Primary Care : Balancing health needs, services, and technology. New York : Oxford University Press; 1998.

16. Wilkinson R, Marmot M (dir.). Social determinants of health. The solid facts ( $2^{\mathrm{e}}$ éd.). Copenhague : World Health Organization; 2003.

17. Sallis JF, Glantz K. Physical activity and food environments : solutions to the obesity epidemic. Milbank Quarterly. 2009; 87:123154.

18. Lemstra M, Neudorf C, Opondo J. Health disparity by neighbourhood income. Revue canadienne de santé publique / Canadian Journal of Public Health. 2006;97:435-439.

19. Krieger J, Higgins DL. Housing and health : time again for public health action. American Journal of Public Health. 2002;92:758-768.
20. Lynch JW, Smith GD, Kaplan GA, House JS. Income inequality and mortality : importance to health of individual income, psychosocial environment, or material conditions. BMJ. 2000;320:1200-1204.

21. Wood D. Effect of child and family poverty on child health in the United States. Pediatrics. 2003;112:707-711.

22. Gidlow C, Cochrane T, Davey RC, Smith G, Fairburn J. Relative importance of physical and social aspects of perceived neighbourhood environment for self-reported health. Preventive Medicine. 2010;51:157-163.

23. Lee DS, Chiu M, Manuel DG et collab. Trends in risk factors for cardiovascular disease in Canada : temporal, socio-demographic and geographic factors. CMAJ 2009;181(3-4):E55-66.

24. Aubé-Maurice J, Rochette L, Blais C. Associations divergentes entre l'incidence de l'hypertension artérielle et la défavorisation selon le mode d'identification des cas. Maladies chroniques et blessures au Canada 2012;32(3):136-146.

25. Campbell NR, McAlister FA, Quan H. Hypertension Outcomes Research Task Force Monitoring and evaluating efforts to control hypertension in Canada : why, how, and what it tells us needs to be done about current care gaps. Canadian Journal of Cardiology. 2013;29(5):564-70.

26. Régie de l'Assurance Maladie du Québec (RAMQ). Banques de données : vue d'ensemble [Internet]. Québec (Qc) : RAMQ; [consultation le 7 juillet 2014]. Consultable à la page : http://www.ramq .gouv.qc.ca/fr/donnees-statistiques/Pages /donnees-statistiques.aspx

27. Ministère de la Santé et des Services Sociaux du Québec (MSSS). Cadre normatif MedÉcho - Mise à jour 2011 [Internet]. Québec (Qc) : MSSS; 2011 [consultation avril 2014]. Consultable à la page : http://msssa4.msss .gouv.qc.ca/fr/document/publication.nsf/0 /581d207c3ed564a7852574bf005283de?Open Document 
28. Statistique Canada. Fichier de conversion des codes postaux (FCCP), guide de référence : code postaux juillet 2009 [Internet]. Ottawa (Ont.) : Statistique Canada; 2010. (n॰92-153G au catalogue). PDF (370 Ko) téléchargeable à partir du lien : www.statcan.gc.ca/pub/92 -153-g/92-153-g2010001-fra.pdf

29. Gamache P, Pampalon R, Hamel D. Guide méthodologique : l'indice de défavorisation matérielle et sociale : en bref. Québec (Qué.) : Institut national de santé publique du Québec; 2010. PDF téléchargeable à partir du lien : http://www2.inspq.qc.ca /santescope/documents/Guide_Metho_Indice_ defavo_Sept_2010.pdf

30. Statistique Canada. Âge (123) et sexe (3) pour la population, pour le Canada, les provinces, les territoires, les divisions de recensement, les subdivisions de recensement et les aires de diffusion, Recensement de 2006 - Données intégrales (100 \%). Ottawa (Ont.) : Statistique Canada; 2007. (n॰ 97-551-X2006006 au catalogue).

31. Kadhim-Saleh A, Green M, Williamson T, Hunter D, Birtwhistle R. Validation of the diagnostic algorithms for 5 chronic conditions in the Canadian Primary Care Sentinel Surveillance Network (CPCSSN): a Kingston Practice-based Research Network (PBRN) report. J Am Board Fam Med. 2013;26(2): 159-67.

32. Lix L, Yogendran M, Burchill C et collab. Defining and validating chronic diseases : an administrative data approach. Winnipeg (Man.) : Manitoba Centre for Health Policy; 2006.

33. Hux JE, Ivis F, Flintoft V, Bica A. Diabetes in Ontario : determination of prevalence and incidence using a validated administrative data algorithm. Diabetes Care. 2002;25(3): 512-6.

34. Naessens JM, Baird MA, Van Houten HK et collab. Predicting persistently high primary care use. Ann Fam Med 2005;3(4):324-30.

35. Stanton MW, Rutherford MK. The high concentration of US health care expenditures. Rockville (MD): Agency for Healthcare Research and Quality; 2005. (Research in Action 19, AHRQ n06-0060).
36. Statistique Canada. Classification des secteurs statistiques (CSS) [Internet]. Ottawa (Ont.) : Statistique Canada [consulté en juillet 2013]. Consultable à la page : http://www12 .statcan.ca/francais/census01/Products /Reference/dict/geo045_f.htm

37. Du Plessis V, Beshiri R, Bollman RD, Clemenson H. Définitions de " rural ». Bulletin d'analyse : Régions rurales et petites villes du Canada. 2001; 3:1-18. PDF (255 Ko) téléchargeable à partir du lien : http://www.statcan.gc.ca/pub/21-006 -x/21-006-x2001003-fra.pdf

38. Pampalon R, Raymond G. Un indice de défavorisation pour la planification de la santé et du bien-être au Québec. Maladies chroniques au Canada. 2000;21(3):104-113.

39. Pampalon R, Hamel D, Gamache P. Recent changes in the geography of social disparities in premature mortality in Quebec. Soc Sci Med. 2008;67:1269-81.

40. Martinez, J, Pampalon R, Hamel, D. Défavorisation et mortalité par accident vasculaire cérébral au Québec. Maladies chroniques au Canada. 2003;24(2-3):62-70.

41. Pampalon R, Raymond G. Indice de défavorisation matérielle et sociale : son application au secteur de la santé et du bien-être. Santé, société et solidarité. 2003;1:191-208.

42. Philibert M, Pampalon R, Hamel D, Thouez JP, Loiselle CG. Material and social deprivation and health and social services utilisation in Quebec : a local-scale evaluation system. Soc Sci Med. 2007;64:1651-64.

43. Carr-Hill RA, Rice N, Roland M. Socioeconomic determinants of rates of consultation in general practice based on fourth national morbidity survey of general practices. BMJ. 1996;312(7037):1008-12.

44. Menec VH, Sirski M, Attawar D. Does continuity of care matter in a universally insured population? Health Serv Res. 2005; 40(2):389-400.

45. Mustard CA, Kaufert P, Kozyrskyj A, Mayer T. Sex differences in the use of health care services. N Engl J Med. 1998;338(23):167883.

46. Bertakis KD, Azari R, Helms LJ, Callahan EJ, Robbins JA. Gender differences in the utilization of health care services. J Fam Pract. 2000;49(2):147-52.
47. Redondo-Sendino A, Guallar-Castillón P, Banegas JR, Rodríguez-Artalejo F. Gender differences in the utilization of health-care services among the older adult population of Spain. BMC Public Health. 2006;16(6):155.

48. Agence de la santé publique du Canada (ASPC). Suivi des maladies du cœur et des accidents vasculaires cérébraux au Canada 2009. Ottawa (Ont.) : ASPC; 2009.

49. Fondation des maladies du cœur du Canada; Société canadienne de cardiologie; Santé Canada. Le fardeau croissant des maladies cardiovasculaires et des accidents vasculaires cérébraux au Canada, 2003. Ottawa (Ont.) : Fondation des maladies du cœur du Canada; 2003.

50. Aubé-Maurice J, Rochette L, Blais C. Relation entre la défavorisation et l'incidence de l'hypertension artérielle chez les individus de 20 ans et plus au Québec en 2006-2007. Québec (Qc) : Institut national de santé publique du Québec; 2010.

51. Matheson FI, White HL, Moineddin R, Dunn JR, Glazier RH. Neighbourhood chronic stress and gender inequalities in hypertension among Canadian adults : a multilevel analysis. J Epidemiol Community Health. 2010;64(8):705-13.

52. Bell AC, Adair LS, Popkin BM. Understanding the role of mediating risk factors and proxy effects in the association between socioeconomic status and untreated hypertension. Soc Sci Med. 2004;59(2):275-83.

53. Hawkley LC, Masi CM, Berry JD, Cacioppo JT. Loneliness is a unique predictor of agerelated differences in systolic blood pressure. Psychol Aging 2006;21(1):152-64.

54. Pears E, Hannaford PC, Taylor MW. Gender, age and deprivation differences in the primary care management of hypertension in Scotland : a cross-sectional database study. Fam Pract. 2003;20(1):22-31.

55. Hammouche S, Holland R, Steel N. Does quality of care for hypertension in primary care vary with postcode area deprivation? An observational study. BMC Health Services Research. 2011;11:297. 
56. Brouwers FM, Courteau J, Grégoire JP et collab. The adequacy of pharmaceutical treatment of schizophrenia in quebec varies with age, but is not influenced by sex or neighbourhood deprivation. Can J Psychiatry. 2013;58(8):456-65.

57. Paré I, Ricard J. Le profil de pratique des médecins omnipraticiens québécois 20062007. Westmount (Qc) : Fédération des médecins omnipraticiens du Québec (FMOQ); 2007. 